\title{
Tropical Australia is a potential reservoir of non-tuberculous mycobacteria in cystic fibrosis
}

\author{
To the Editor:
}

Improved survival rates and increased treatment intensity of people with cystic fibrosis have been accompanied by a rising incidence of multi-antibiotic resistant and difficult-to-treat respiratory pathogens, including non-tuberculous mycobacteria (NTM) [1]. NTM epidemiology in cystic fibrosis varies globally, with a prevalence of $>20 \%$ in some geographical locations [2]. In particular, there are concerns that active NTM disease from rapidly growing mycobacteria (Mycobacterium abscessus complex) may be increasing and causing accelerated pulmonary decline $[3,4]$. NTM are found naturally in ecological niches such as soil and water, and susceptible individuals may also acquire infection from potable water in their homes $[5,6]$. Recent reports demonstrate person-to-person transmission $[7,8]$, which might occur via fomites and cough aerosols [8] and further emphasise the potential clinical importance of these organisms.

The principal aim of this observational study was to determine predictors of NTM acquisition in the adult cystic fibrosis patient cohort at The Prince Charles Hospital (TPCH) in Queensland, Australia. The study was approved by TPCH Human and Research Ethics Committee, Metro North Hospital and Health Service, Queensland, Australia (HREC/13/QPCH/51).

Patients with cystic fibrosis $(n=434)$ receiving their care at TPCH between 2001-2013 were included. Patients were stratified into one of two cohorts depending on whether they were NTM-positive or -negative (naïve) based on sputum culture results, which were available for 399 out of 434 (92\%) patients. The remaining patients $(n=35)$ who were not screened for NTM during the study period were classed as NTM-naïve. Mycobacterial culture and species identification methods have been described previously [9]. The date of NTM acquisition was defined as the date of the first positive culture identified by the Queensland Mycobacterial Reference Laboratory or the referring cystic fibrosis centre (if the patient was transferred to TPCH). Active NTM disease was defined according to American Thoracic Society guidelines [10].

Clinical and demographic data (table 1) were gathered from medical records, the referring cystic fibrosis centre or the Australian Cystic Fibrosis Data Registry. For NTM-positive patients, these data correlated with the date of NTM acquisition. In NTM-naïve patients, data corresponded to the calendar year prior to January 1,2013 , or the year prior to death $(n=43)$ or lung transplantation $(n=72)$. Data could not be retrieved for NTM-naïve patients who had not attended the clinic during 2012 (previously moved interstate/overseas or lost to follow-up).

TPCH is uniquely situated geographically with a catchment area of 1.8 million $\mathrm{km}^{2}$, including zones north (tropical) and south (sub-tropical) of the Tropic of Capricorn (latitude $23.5^{\circ}$ south of the equator). Therefore, the residential location for each person was defined as tropical or sub-tropical according to residence area in the 5 years prior to 1$)$ the date of NTM acquisition, or 2) January 1, 2013, death ( $n=43)$, or lung transplantation $(n=72)$, if NTM-naïve. Patients who had resided in both zones during these 5 years were excluded from further analyses $(n=9)$.

Categorical data were analysed using a Chi-squared test with Yates' continuity correction or Fisher's exact test, as appropriate. Univariate and multivariate logistic regression analysis was performed to identify factors associated with NTM acquisition based on complete data from 375 patients. Variables with $\mathrm{p}<0.1$ were included in the multivariable model. Cox regression survival analysis was used to assess the association

@ERSpublications

Living in tropical Australia is associated with NTM acquisition, whilst long-term azithromycin is protective in CF http://ow.ly/FrJi309W8DK

Cite this article as: Sherrard LJ, Tay GT, Butler CA, et al. Tropical Australia is a potential reservoir of non-tuberculous mycobacteria in cystic fibrosis. Eur Respir J 2017; 49: 1700046 [https://doi.org/10.1183/ 13993003.00046-2017]. 


\begin{tabular}{|c|c|c|}
\hline & Odds ratio $(95 \% \mathrm{Cl})$ & p-value \\
\hline \multicolumn{3}{|l|}{ Univariate model } \\
\hline Age (per 10 years) & $0.7(0.5-0.9)$ & 0.02 \\
\hline Gender & $1.0(0.6-1.8)$ & 1.0 \\
\hline \multicolumn{3}{|l|}{ CFTR function $\#$} \\
\hline Residual versus minimal & $1.3(0.5-3.8)$ & 0.6 \\
\hline Non-classified versus minimal & $0.6(0.3-1.2)$ & 0.2 \\
\hline Tropical residence in the previous 5 years & $2.9(1.5-5.4)$ & 0.001 \\
\hline Chronic Pseudomonas aeruginosa infection & $0.6(0.3-1.4)$ & 0.2 \\
\hline FEV $1 \%$ pred (per $10 \%$ increase) & $1.2(1.1-1.4)$ & $<0.001$ \\
\hline Pancreatic sufficiency status & $0.5(0.2-1.0)$ & 0.04 \\
\hline Not prescribed azithromycin for $>6$ months in the preceding year & $15.7(8.1-30.6)$ & $<0.001$ \\
\hline Hospital admissions in the preceding year (per five admissions) & $0.3(0.1-0.7)$ & 0.003 \\
\hline Hospital days in the preceding year (per 10 hospital days) & $0.8(0.7-0.9)$ & 0.002 \\
\hline \multicolumn{3}{|l|}{ Multivariate model ${ }^{\pi}$} \\
\hline Age (per 10 years) & $0.7(0.5-1.0)$ & 0.03 \\
\hline Tropical residence in the previous 5 years & $2.5(1.2-5.4)$ & 0.02 \\
\hline Not prescribed azithromycin for $>6$ months in the preceding year & $15.4(7.8-30.5)$ & $<0.001$ \\
\hline \multicolumn{3}{|c|}{$\begin{array}{l}\text { CFTR: cystic fibrosis transmembrane conductance regulator; } F E V_{1} \text { : forced expiratory volume in } 1 \mathrm{~s} \text {. } \\
\# \text { : definitions of CFTR function: residual function, harbouring } \geqslant 1 \text { allele with class IV-V mutations; minimal } \\
\text { function, harbouring two alleles with class I-III mutations; non-classified, harbouring two alleles with } \\
\text { mutations of unknown function. १: final model shows predictor variables that retained a significant } \\
\text { association with NTM acquisition after adjustment for all other factors. }\end{array}$} \\
\hline
\end{tabular}

between NTM acquisition and active NTM disease (both modelled as time-dependent covariates) and time to death or lung transplantation (based on the entire cystic fibrosis cohort with all data censored to December 2013). Data were analysed using Stata v14 (StataCorp) or SPSS v22 software (IBM).

Spatial clustering of NTM has been reported in the USA, with western and south-eastern states designated as high-risk areas and associated with high surface water and atmospheric moisture availability [11]. In our study, NTM was detected in 54 out of 375 (14\%) patients with cystic fibrosis. In a multivariate logistic regression, the odds of NTM acquisition were 2.5 (95\% CI 1.2-5.4) times higher (table 1) in those who resided in a tropical zone $(n=19 / 70,27 \%)$ than in those who resided in a sub-tropical zone $(n=35 / 305,11 \%)$.

Factors such as heightened clinician awareness, increased surveillance practices, and improved culture procedures have likely contributed to increased detection and identification of NTM $[4,12]$. At TPCH, the percentage of sputum samples screened for NTM increased by $>60 \%$ between 2001 and 2013 . The mean number of sputum samples per person that underwent NTM screening was similar between those patients living in tropical zones (2.8 samples per person per year) and those living in sub-tropical zones (2.5 samples/person/year).

Mycobacterial species identification was available for 72 out of 73 (99\%) isolates, and a quarter of NTM-positive patients acquired more than one NTM species during the study period (tropical patients, $\mathrm{n}=6 / 70,9 \%$; sub-tropical patients, $\mathrm{n}=7 / 305,2 \% ; \mathrm{p}=0.02$ ) with a range of one to four species identified per person, potentially adding to the complexity when selecting treatment. The most commonly identified NTM species were $M$. abscessus complex $(\mathrm{n}=33 / 73,45 \%)$ and the slow-growing species Mycobacterium intracellulare ( $\mathrm{n}=23 / 73,32 \%)$. Previously, spatial clusters of these NTM species were found in Queensland (M. abscessus complex, coastal Whitsunday region, tropical zone; M. intracellulare, agricultural Darling Downs region, sub-tropical zone) [9]. When the primary NTM species acquired was considered for each cystic fibrosis patient, an association between $M$. abscessus complex and geographical zone was observed $(\mathrm{p}=0.02)$, with a higher recovery from tropical patients $(\mathrm{n}=10 / 70,14 \%)$ than from sub-tropical patients $(\mathrm{n}=17 / 305,6 \%)$. Conversely, no association was found between $M$. intracellulare acquisition and geographical zone (tropical patients, $n=6 / 70,9 \%$; sub-tropical patients, $n=10 / 305,3 \%$; $=0.09$ ). Further studies are required to determine the specific regions within each zone that are at high risk for acquisition of a particular NTM species in cystic fibrosis. Other rapidly growing species identified were Mycobacterium chelonae $(\mathrm{n}=2)$ and Mycobacterium fortuitum $(\mathrm{n}=3)$, and the remaining slow-growing species included Mycobacterium avium (n=6), Mycobacterium scrofulaceum ( $\mathrm{n}=2)$, Mycobacterium lentiflavum ( $\mathrm{n}=1)$, Mycobacterium simiae $(\mathrm{n}=1)$ and Mycobacterium shimoidei $(\mathrm{n}=1)$. 
NTM lung disease was reported to occur in $69 \%$ of $M$. abscessus-infected cystic fibrosis patients [4]. Here, active NTM disease was diagnosed in 14 out of 70 (20\%) tropical patients and 11 out of $305(4 \%)$ sub-tropical patients $(\mathrm{p}<0.001)$, and overall, 23 out of $25(92 \%)$ people diagnosed with lung disease tested positive for $M$. abscessus complex.

Markers for severe cystic fibrosis disease (e.g. pancreatic insufficiency, minimal CFTR (cystic fibrosis transmembrane conductance regulator) function, Pseudomonas aeruginosa infection) have also been reported to coincide with higher rates of NTM infection [4]. Whilst we did not find such markers to be associated with NTM acquisition (table 1) after adjustment for other variables, we found that macrolide treatment for at least 6 months in the preceding year was associated with reduced NTM acquisition. The adjusted odds ratio of NTM acquisition was 15.4 (95\% CI 7.8-30.5) times higher in those who were not prescribed azithromycin than in those who were prescribed it (table 1). The role of macrolide antibiotic use in NTM infection remains controversial, with one study suggesting enhanced risk of NTM, and others refuting this claim [13-15].

The impact of NTM infection and patient survival is currently unclear. One study observed that a quarter of patients with $M$. abscessus complex died or were lung transplanted [12]. Here, neither being NTM positive (hazards ratio $0.6,95 \%$ CI $0.2-1.4 ; \mathrm{p}=0.2$ ) nor being diagnosed with active NTM disease (hazards ratio $0.6,95 \%$ CI $0.2-2.6 ; \mathrm{p}=0.5$ ) was associated with an increased risk of death/lung transplantation.

Although this study was limited by its retrospective design, we found that living in the tropical zone of Australia was independently associated with NTM acquisition and that long-term azithromycin treatment was protective. Tropical patients were more likely to acquire more than one NTM species and have a diagnosis of active NTM disease. Australian population-based studies are required to determine specific host, socio-ecological, economic, climatic and environmental factors that affect the acquisition and development of active NTM disease in cystic fibrosis.

Laura J. Sherrard ${ }^{1,7}$, George T. Tay ${ }^{2,7}$, Claire A. Butler ${ }^{2}$, Michelle E. Wood ${ }^{1,2,3}$, Stephanie Yerkovich $^{3}$, Kay A. Ramsay ${ }^{1,3}$, David W. Reid ${ }^{2,4}$, Vanessa L. Moore ${ }^{2}$, Timothy J. Kidd ${ }^{5,6}$ and Scott C. Bell ${ }^{1,2,3}$

${ }^{1}$ Lung Bacteria Group, QIMR Berghofer Medical Research Institute, Brisbane, Australia. ${ }^{2}$ Adult Cystic Fibrosis Centre, The Prince Charles Hospital, Brisbane, Australia. ${ }^{3}$ School of Medicine, The University of Queensland, Brisbane, Australia. ${ }^{4}$ Lung Inflammation and Infection Group, QIMR Berghofer Medical Research Institute, Brisbane, Australia. ${ }^{5}$ School of Chemistry and Molecular Biosciences, The University of Queensland, Brisbane, Australia. ${ }^{6}$ Centre for Experimental Medicine, Queen's University Belfast, Belfast, UK. ${ }^{7}$ These authors contributed equally to this article.

Correspondence: Laura J. Sherrard, QIMR Berghofer Medical Research Institute, 300 Herston Road, Herston, Brisbane, QLD 4006, Australia. E-mail: 1sherrard03@qub.ac.uk

Received: May 192016 | Accepted after revision: Feb 132017

Support statement: This study was funded by the Department of Health, Australian Government, National Health and Medical Research Council (APP 102494). Funding information for this article has been deposited with the Crossref Funder Registry.

Conflict of interest: Disclosures can be found alongside this article at erj.ersjournals.com

\section{References}

1 Cystic Fibrosis Foundation Patient Registry. 2014 Annual Data Report. Bethesda, Cystic Fibrosis Foundation, 2015.

2 Adjemian J, Olivier KN, Prevots DR. Nontuberculous mycobacteria among patients with cystic fibrosis in the United States: screening practices and environmental risk. Am J Respir Crit Care Med 2014; 190: 581-586.

3 Esther CR Jr, Esserman DA, Gilligan P, et al. Chronic Mycobacterium abscessus infection and lung function decline in cystic fibrosis. J Cyst Fibros 2010; 9: 117-123.

4 Bar-On O, Mussaffi $\mathrm{H}$, Mei-Zahav $\mathrm{M}$, et al. Increasing nontuberculous mycobacteria infection in cystic fibrosis. J Cyst Fibros 2015; 14: 53-62.

5 Thomson R, Tolson C, Carter R, et al. Isolation of nontuberculous mycobacteria (NTM) from household water and shower aerosols in patients with pulmonary disease caused by NTM. J Clin Microbiol 2013; 51: 3006-3011.

6 Prevots DR, Adjemian J, Fernandez AG, et al. Environmental risks for nontuberculous mycobacteria. Individual exposures and climatic factors in the cystic fibrosis population. Ann Am Thorac Soc 2014; 11: 1032-1038.

7 Bryant JM, Grogono DM, Greaves D, et al. Whole-genome sequencing to identify transmission of Mycobacterium abscessus between patients with cystic fibrosis: a retrospective cohort study. Lancet 2013; 381: 1551-1560.

8 Bryant JM, Grogono DM, Rodriguez-Rincon D, et al. Emergence and spread of a human-transmissible multidrug-resistant nontuberculous mycobacterium. Science 2016; 354: 751-757.

9 Chou MP, Clements AC, Thomson RM. A spatial epidemiological analysis of nontuberculous mycobacterial infections in Queensland, Australia. BMC Infect Dis 2014; 14: 279.

10 Griffith DE, Aksamit T, Brown-Elliott BA, et al. An official ATS/IDSA statement: diagnosis, treatment, and prevention of nontuberculous mycobacterial diseases. Am J Respir Crit Care Med 2007; 175: 367-416.

11 Adjemian J, Olivier KN, Seitz AE, et al. Spatial clusters of nontuberculous mycobacterial lung disease in the United States. Am J Respir Crit Care Med 2012; 186: 553-558.

12 Qvist T, Gilljam M, Jonsson B, et al. Epidemiology of nontuberculous mycobacteria among patients with cystic fibrosis in Scandinavia. J Cyst Fibros 2015; 14: 46-52. 
13 Renna M, Schaffner C, Brown K, et al. Azithromycin blocks autophagy and may predispose cystic fibrosis patients to mycobacterial infection. J Clin Invest 2011; 121: 3554-3563.

14 Coolen N, Morand P, Martin C, et al. Reduced risk of nontuberculous mycobacteria in cystic fibrosis adults receiving long-term azithromycin. J Cyst Fibros 2015; 14: 594-599.

15 Binder AM, Adjemian J, Olivier KN, et al. Epidemiology of nontuberculous mycobacterial infections and associated chronic macrolide use among persons with cystic fibrosis. Am J Respir Crit Care Med 2013; 188: 807-812.

Copyright (C)ES 2017 